\title{
Two type quasi-contractions on quasi metric spaces and some fixed point results
}

\author{
Hakan Şimşek ${ }^{a, *}$, Ishak Altun ${ }^{a, b}$ \\ ${ }^{a}$ Department of Mathematics, Faculty of Science and Arts, Kirikkale University, 71450 Yahsihan, Kirikkale, Turkey. \\ ${ }^{b}$ King Saud University, College of Science, Riyadh, Saudi Arabia.
}

Communicated by M. Imdad

\begin{abstract}
In this paper, we introduce new concepts of quasi-contractions of type (A) and of type (B) in a quasi metric space and we present the differences between of them. Then we present some fixed point results. In the light of the theorems it is shown that, although the Hausdorffness condition of quasi metric space is needed for the mapping of quasi contraction of type (A), it is not necessary to guarantee the existence of fixed point for the mapping of quasi contraction of type (B). C)2017 All rights reserved.
\end{abstract}

Keywords: Quasi metric space, left K-Cauchy sequence, left $\mathcal{K}$-completeness, fixed point, quasi contraction. 2010 MSC: $54 \mathrm{H} 25,47 \mathrm{H} 10$.

\section{Introduction and preliminaries}

Let $X$ be any set. Then a function $d: X \times X \rightarrow[0, \infty)$ is said to be a quasi metric on $X$ if it has the following properties for all $x, y, z \in X$;

- $\mathrm{d}(x, x)=0$;

- $d(x, y) \leqslant d(x, z)+d(z, y)$

- $d(x, y)=d(y, x)=0 \Rightarrow x=y$.

If a quasi metric d satisfies the additional condition

- $d(x, y)=0 \Rightarrow x=y$,

then $d$ is said to be $T_{1}$-quasi metric. It is clear that, every metric is a $T_{1}$-quasi metric, every $T_{1}$-quasi metric is a quasi metric. A quasi (resp. $T_{1}$-quasi) metric space is a pair $(X, d)$ such that $X$ is a nonempty set and $d$ is a quasi (resp. $T_{1}$-quasi) metric. Given a quasi metric space $(X, d)$ and any real number $\varepsilon>0$, the open

\footnotetext{
*Corresponding author

Email addresses: hsimsek@kku.edu.tr (Hakan Şimşek), ishakaltun@yahoo.com (Ishak Altun)
} 
ball and closed ball, respectively, of radius $\varepsilon$ and center $x_{0} \in X$ is the set $B_{d}\left(x_{0}, \varepsilon\right) \subset X$ and $B_{d}\left[x_{0}, \varepsilon\right] \subset X$ defined by

$$
B_{d}\left(x_{0}, \varepsilon\right)=\left\{y \in X: d\left(x_{0}, y\right)<\varepsilon\right\},
$$

and

$$
B_{d}\left[x_{0}, \varepsilon\right]=\left\{y \in X: d\left(x_{0}, y\right) \leqslant \varepsilon\right\} .
$$

Every quasi metric $d$ on $X$ generates a natural topology on $X$. This topology on $X$ generated by the family of open balls $\left\{B_{d}(x, \varepsilon): x \in X\right.$ and $\left.\varepsilon>0\right\}$ as base is called the the topology $\tau_{d}$ (or, the topology induced by the quasi metric $d)$. If $(X, d)$ is a quasi metric space, then $\tau_{d}$ is a $T_{0}$ topology, and if $(X, d)$ is a $T_{1}$-quasi metric space, then $\tau_{d}$ is a $T_{1}$ topology. It is clear that if $d$ is a quasi metric and $\tau_{d}$ is $T_{1}$ topology, then $d$ is $T_{1}$-quasi metric. However, there exist some examples of $T_{1}$ topological spaces which are not $T_{1}$-quasi metrizable. Uncountable cofinite topological space, which is not first countable space, can be given an example to this situation.

Given a quasi metric $d$ on $X$, the function $d^{-1}$ defined by for all $x, y \in X$,

$$
d^{-1}(x, y)=d(y, x)
$$

is also a quasi metric on $X$ and the functions $d^{s}$ and $d_{+}$defined by for all $x, y \in X$,

$$
d^{s}(x, y)=\max \left\{d(x, y), d^{-1}(x, y)\right\}
$$

and

$$
d_{+}(x, y)=d(x, y)+d^{-1}(x, y)
$$

are metrics on $X$. Moreover, metrics $d^{s}$ and $d_{+}$are equivalent. Let $\left\{x_{n}\right\}$ be a sequence in the quasi metric space $(X, d)$ and $a \in X$. The sequence $\left\{x_{n}\right\}$ is said to be convergent to $a$ with respect to $\tau_{d}$ if $d\left(a, x_{n}\right) \rightarrow 0$ as $n \rightarrow \infty$. This is called as d-convergence and denoted by the following

$$
x_{\mathrm{n}} \stackrel{\mathrm{d}}{\rightarrow} \mathrm{a} \Leftrightarrow \mathrm{d}\left(\mathrm{a}, x_{\mathrm{n}}\right) \rightarrow 0 .
$$

Similarly, the sequence $\left\{x_{n}\right\}$ is said to be convergent to a with respect to $\tau_{d^{-1}}$ if $d\left(x_{n}, a\right) \rightarrow 0$ as $n \rightarrow \infty$. This is called as $\mathrm{d}^{-1}$-convergence and denoted by the following

$$
x_{n} \stackrel{d^{-1}}{\rightarrow} a \Leftrightarrow d\left(x_{n}, a\right) \rightarrow 0 .
$$

Finally, the sequence $\left\{x_{n}\right\}$ is said to be convergent to a with respect to $\tau_{d^{s}}$ if $d^{s}\left(a, x_{n}\right) \rightarrow 0$ as $n \rightarrow \infty$. This is called as $\mathrm{d}^{\mathrm{s}}$-convergence and denoted by the following

$$
x_{n} \stackrel{d^{s}}{\rightarrow} a \Leftrightarrow d^{s}\left(a, x_{n}\right) \rightarrow 0
$$

Thus, it is clear that $x_{n} \stackrel{d^{s}}{\rightarrow} a \Leftrightarrow x_{n} \stackrel{d}{\rightarrow} a$ and $x_{n} \stackrel{d^{-1}}{\rightarrow} a$. More detailed information for some important properties of quasi metric spaces and their topological structures can be seen in $[4,7,8]$.

There exist several different notions of Cauchy sequence and of completeness in the quasi metric version of studies of fixed point theory. In fact, there are seven different definitions of Cauchy sequence on quasi metric spaces. Also, taking into account three different convergence of a sequence in quasi metric spaces, there obtain twenty one different notions of completeness. We can find more detailed information about some kind of convergence, Cauchyness, completeness and some important properties of quasi metric space in [1,2,9-11]. In this present paper, we will denote by $\mathbb{R}$ the set of all real numbers and by $\mathbb{N}$ the set of all natural numbers and also we shall use the following notions.

A sequence $\left\{x_{n}\right\}$ in a quasi metric space $(X, d)$ is called

- left K-Cauchy if for every $\varepsilon>0$, there exists $n_{0} \in \mathbb{N}$ such that

$$
\forall n, k, \quad n \geqslant k \geqslant n_{0}, \quad d\left(x_{k}, x_{n}\right)<\varepsilon ;
$$


- right K-Cauchy if for every $\varepsilon>0$, there exists $\mathrm{n}_{0} \in \mathbb{N}$ such that

$$
\forall n, k, \quad n \geqslant k \geqslant n_{0}, \quad d\left(x_{n}, x_{k}\right)<\varepsilon ;
$$

- $d^{s}$-Cauchy if for every $\varepsilon>0$, there exists $n_{0} \in \mathbb{N}$ such that

$$
\forall \mathrm{n}, \mathrm{k} \geqslant \mathrm{n}_{0}, \quad \mathrm{~d}\left(\mathrm{x}_{\mathrm{n}}, \mathrm{x}_{\mathrm{k}}\right)<\varepsilon .
$$

It is obvious that if a sequence is left K-Cauchy with respect to $\mathrm{d}$, then it is right $\mathrm{K}$-Cauchy with respect to $\mathrm{d}^{-1}$ and $\left\{x_{n}\right\}$ is $\mathrm{d}^{\mathrm{s}}$-Cauchy if and only if it is both left K-Cauchy and right K-Cauchy. If a sequence $\left\{x_{n}\right\}$ in a quasi metric space $(X, d)$ satisfies $\sum_{n=1}^{\infty} d\left(x_{n}, x_{n+1}\right)<\infty$, then it is left K-Cauchy sequence (see [4]). In a quasi metric space, $\mathrm{d}$ or $\mathrm{d}^{-1}$-convergent sequences may not be Cauchy in the sense mentioned above, although every convergent sequence is Cauchy in a metric space (see [9]).

A quasi metric space $(X, d)$ called

- bicomplete if every $\mathrm{d}^{\mathrm{s}}$-Cauchy sequence is $\mathrm{d}^{\mathrm{s}}$-convergent;

- left (right) $\mathcal{K}$-complete if every left (right) K-Cauchy sequence is d-convergent;

- left (right) $\mathcal{M}$-complete if every left (right) K-Cauchy sequence is $\mathrm{d}^{-1}$-convergent;

- left (right) Smyth complete if every left (right) K-Cauchy sequence is $\mathrm{d}^{\mathrm{s}}$-convergent.

If $(\mathrm{X}, \mathrm{d})$ is both left $\mathcal{K}$-complete and left $\mathcal{M}$-complete, then it is bicomplete. The same implications hold for the notions of right completeness.

The aim of this paper is to present several new fixed point results for quasi contractive mappings on some kind of complete in quasi metric spaces. It can be found some interesting and nice fixed point results on quasi metric spaces in $[5,6,12]$.

\section{Fixed point results}

Definition 2.1. Let $(X, d)$ be a quasi metric space and $T: X \rightarrow X$ be a mapping. Then $T$ is said to be

- quasi contraction of type (A), if there exists $\lambda \in(0,1)$ such that

$$
d(T x, T y) \leqslant \lambda M_{A}(x, y)
$$

for all $x, y \in X$, where

$$
M_{A}(x, y)=\max \{d(x, y), d(x, T x), d(y, T y), d(x, T y), d(y, T x)\}
$$

- quasi contraction of type (B), if there exists $\lambda \in(0,1)$ such that

$$
d(T x, T y) \leqslant \lambda M_{B}(x, y)
$$

for all $x, y \in X$, where

$$
M_{B}(x, y)=\max \{d(y, x), d(x, T x), d(y, T y), d(T y, x), d(y, T x)\} .
$$

Example 2.2. Let $X=\{0,1,2, \cdots\}$ and $d: X \times X \rightarrow \mathbb{R}$ be a quasi metric on $X$ given by

$$
\begin{array}{rlrl}
\mathrm{d}(\mathrm{n}, \mathrm{n}) & =0, & & \forall \mathrm{n} \in \mathrm{X}, \\
\mathrm{d}(0, \mathrm{n}) & =\mathrm{d}(\mathrm{n}, 1)=0, & \forall \mathrm{n} \geqslant 1, \\
\mathrm{~d}(\mathrm{n}, 0) & =1, & \forall \mathrm{n} \geqslant 1, \\
\mathrm{~d}(\mathrm{n}, \mathrm{m}) & =\frac{1}{2^{\mathrm{n}+1}}+\frac{1}{2^{\mathrm{m}+1}}, & \text { otherwise. }
\end{array}
$$

Now define $T: X \rightarrow X$ by $T(n)=n+1$ for all $n \in X$. Then $T$ is quasi contraction of type (B) but not of 
type (A).

Proposition 2.3. Let $(\mathrm{X}, \mathrm{d})$ be a quasi-metric space. If $\mathrm{T}$ is a quasi contraction of type $(A)$ or of type $(B)$ on $(\mathrm{X}, \mathrm{d})$, then it is a quasi contraction on the metric space $\left(X, \mathrm{~d}^{\mathrm{s}}\right)$.

Proof. Suppose $T$ is a quasi contraction of type (A) on $(X, d)$. Then, there exists $\lambda \in(0,1)$ such that

$$
d(T x, T y) \leqslant \lambda M_{A}(x, y)
$$

for all $x, y \in X$. Thus, given $x, y \in X$, we also have

$$
d(T y, T x) \leqslant \lambda M_{A}(y, x)
$$

So, we obtain

$$
d^{s}(T x, T y) \leqslant \lambda \max \left\{d^{s}(x, y), d^{s}(x, T x), d^{s}(y, T y), d^{s}(x, T y), d^{s}(y, T x)\right\}
$$

That is, $T$ is a quasi contraction on $\left(X, d^{s}\right)$. Similarly, we show that if $T$ is a quasi contraction of type (B) on $(X, d)$, then it is a quasi contraction on $\left(X, d^{s}\right)$.

Proposition 2.4. Let $(\mathrm{X}, \mathrm{d})$ be a quasi metric space. If $\mathrm{T}$ is a quasi contraction of type $(A)$ or of type $(B)$ on $(\mathrm{X}, \mathrm{d})$, then the Picard sequence $\left\{x_{n}\right\}$ generated by $T$ with initial value $x_{0} \in X$, where $x_{n}=T x_{n-1}$ for all $n \in \mathbb{N}$, is a $\mathrm{d}^{\mathrm{s}}$-Cauchy sequence on $(X, \mathrm{~d})$.

Proof. By Proposition 2.3, T is a quasi contraction on $\left(X, \mathrm{~d}^{\mathrm{s}}\right)$. Then, from the proof of [3, Theorem 4.12] we conclude that $\left\{x_{n}\right\}$ is a Cauchy sequence in $\left(X, d^{s}\right)$. Therefore it is $d^{s}$-Cauchy sequence on $(X, d)$.

Theorem 2.5. Let $(X, d)$ be a bicomplete quasi metric space and $\mathrm{T}: \mathrm{X} \rightarrow \mathrm{X}$ be a quasi contraction of type $(A)$ or of type (B). Then $\mathrm{T}$ has a unique fixed point.

Proof. Since $(X, d)$ is bicomplete quasi metric space, then $\left(X, d^{s}\right)$ is complete metric space. By Proposition 2.3, $T$ is a quasi contraction on $\left(X, d^{s}\right)$. Then, from the proof of Theorem 4.12 in [3] we conclude that $T$ has a unique fixed point in $X$.

In the following, we provide some fixed point results considering more weak completeness instead of bicompleteness of $(X, d)$.

Proposition 2.6. Let $(\mathrm{X}, \mathrm{d})$ be a $\mathrm{T}_{1}$-quasi metric space. If $\mathrm{T}$ is a quasi contraction of type $(A)$ or of type $(B)$ on $(\mathrm{X}, \mathrm{d})$, then $\mathrm{T}$ has at most one fixed point.

Proof. Let $\mathrm{T}$ be the quasi contraction of type (A) and let T has two different fixed points, say $z, w \in X$. Since $d$ is a $T_{1}$-quasi metric $\min \{d(z, w), d(w, z)\}>0$. Now if $d(w, z) \leqslant d(z, w)$, then we get

$$
\begin{aligned}
0 & <\mathrm{d}(z, w)=\mathrm{d}(\mathrm{T} z, \mathrm{~T} w) \\
& \leqslant \lambda M_{A}(z, w) \\
& =\lambda \max \{\mathrm{d}(z, w), \mathrm{d}(z, \mathrm{~T} z), \mathrm{d}(w, \mathrm{~T} w), \mathrm{d}(z, \mathrm{~T} w), \mathrm{d}(w, \mathrm{~T} z)\} \\
& =\lambda \mathrm{d}(z, w),
\end{aligned}
$$

which is a contradiction. If, $\mathrm{d}(w, z)>\mathrm{d}(z, w)$, then we get

$$
\begin{aligned}
0 & <\mathrm{d}(w, z)=\mathrm{d}(\mathrm{T} w, \mathrm{~T} z) \\
& \leqslant \lambda M_{A}(w, z) \\
& =\lambda \max \{\mathrm{d}(w, z), \mathrm{d}(w, \mathrm{~T} w), \mathrm{d}(z, \mathrm{~T} z), \mathrm{d}(w, \mathrm{~T} z), \mathrm{d}(z, \mathrm{~T} w)\} \\
& =\lambda \mathrm{d}(w, z),
\end{aligned}
$$

which is a contradiction. Therefore $T$ has at most one fixed point. Similar result can be obtained when $T$ is a quasi contraction of type (B). 
We will consider the sequential continuity of a mapping $T$ in our theorems.

Definition 2.7. Let $X$ be a nonempty set, $d$ and $\rho$ be two quasi metrics on $X$ and $T: X \rightarrow X$ be a mapping. Then $T$ is said to be sequentially $d$ - $\rho$-continuous at $x \in X$, if for all sequence $\left\{x_{n}\right\}$ in $X$ such that $d\left(x, x_{n}\right) \rightarrow 0$ implies $\rho\left(T x, T x_{n}\right) \rightarrow 0$. If $T$ is sequentially $d$ - $\rho$-continuous at all points of $X$, then $T$ is said to be sequentially $d-\rho$-continuous on $X$.

Our first main results is as follows:

Theorem 2.8. Let $(\mathrm{X}, \mathrm{d})$ be a left $\mathcal{K}$-complete $\mathrm{T}_{1}$-quasi metric space and $\mathrm{T}: \mathrm{X} \rightarrow \mathrm{X}$ be a quasi contraction of type (A). Then, $\mathrm{T}$ has a unique fixed point in $\mathrm{X}$ provided that one of the following conditions holds:

(i) $\left(\mathrm{X}, \tau_{\mathrm{d}}\right)$ is Hausdorff space and $\mathrm{T}$ is sequentially $\mathrm{d}$-d-continuous;

(ii) $\mathrm{T}$ is sequentially $\mathrm{d}-\mathrm{d}^{-1}$-continuous;

(iii) Thas the following property:

$$
\inf \{d(y, x)+d(x, T x): x \in X\}>0
$$

for every $y \in X$ with $y \neq T y$.

Proof. Let $x_{0} \in X$ be arbitrary and $\left\{x_{n}\right\}$ be the Picard sequence, that is, $x_{n}=T x_{n-1}$ for all $n \in \mathbb{N}$. By Proposition 2.4, the sequence $\left\{x_{n}\right\}$ is a left K-Cauchy sequence in the quasi metric space $(X, d)$. Then, since $(X, d)$ is left $\mathcal{K}$-complete, there exists $z \in X$ such that $\left\{x_{n}\right\}$ is d-converges to $z$, that is, $d\left(z, x_{n}\right) \rightarrow 0$ as $\mathrm{n} \rightarrow \infty$.

Now, we will consider the following three cases:

(i) Let $\left(X, \tau_{d}\right)$ be Hausdorff space and $T$ be sequentially $d$-d-continuous mapping. Then

$$
\mathrm{d}\left(\mathrm{T} z, x_{\mathrm{n}+1}\right)=\mathrm{d}\left(\mathrm{T} z, \mathrm{~T} x_{\mathrm{n}}\right) \rightarrow 0 \text { as } \mathrm{n} \rightarrow \infty .
$$

Since $(X, d)$ is Hausdorff space, we get $z=T z$.

(ii) Let $T$ be sequentially $d-d^{-1}$-continuous. Then, since $d\left(z, x_{n}\right) \rightarrow 0$ as $n \rightarrow \infty$, we get

$$
\mathrm{d}\left(T x_{n}, T z\right)=d^{-1}\left(T z, T x_{n}\right) \rightarrow 0 \text { as } n \rightarrow \infty .
$$

So, we obtain

$$
d(z, T z) \leqslant d\left(z, x_{n+1}\right)+d\left(T x_{n}, T z\right) \rightarrow 0 \text { as } n \rightarrow \infty .
$$

Since $d$ is $T_{1}$-quasi metric, we get $z=T z$.

(iii) Let $T$ has a property that $\inf \{d(y, x)+d(x, T x): x \in X\}>0$ for every $y \in X$ with $y \neq T y$. Now, suppose that $z \neq \mathrm{T} z$, then by our assumption, we obtain (by taking on account the left K-Cauchyness of $\left.\left\{x_{n}\right\}\right)$

$$
\begin{aligned}
0 & <\inf \{d(z, x)+d(x, T x): x \in X\} \\
& \leqslant \inf \left\{d\left(z, x_{n}\right)+d\left(x_{n}, T x_{n}\right): n \in \mathbb{N}\right\} \\
& =\inf \left\{d\left(z, x_{n}\right)+d\left(x_{n}, x_{n+1}\right): n \in \mathbb{N}\right\} \\
& =0
\end{aligned}
$$

which is a contradiction. Hence $z=T z$. By Proposition 2.6, the fixed point is unique.

The following example shows that the Hausdorffness condition can not be removed in Theorem 2.8. 
Example 2.9. Let $X=\{1,2,3, \cdots\}$ and $d$ be a quasi metric on $X$ given by $d(n, n)=0$ for all $n \in X$, and $d(n, m)=\frac{1}{m}$ otherwise. Then $d$ is a $T_{1}$-quasi metric on $X$ however $\left(X, \tau_{d}\right)$ is not Hausdorff. Since every left $\mathrm{K}$-Cauchy sequence $d$-converges to 1 , then $(X, d)$ is left $\mathcal{K}$-complete quasi metric space. Now we define $\mathrm{T}: X \rightarrow X$ as $\mathrm{Tn}=2 \mathrm{n}$ for all $\mathrm{n} \in \mathrm{X}$, then $\mathrm{T}$ is a quasi contraction of type (A) with $\lambda=\frac{1}{2}$. Further $\mathrm{T}$ is sequentially $d$-d-continuous. However $T$ has no fixed point. (Note that both (ii) and (iii) conditions are not satisfied)

If we consider the quasi contraction of type (B) instead of type (A), we do not need the Hausdorffness condition of $(X, d)$.

Theorem 2.10. Let $(\mathrm{X}, \mathrm{d})$ be a left $\mathcal{K}$-complete $\mathrm{T}_{1}$-quasi metric space and $\mathrm{T}: \mathrm{X} \rightarrow \mathrm{X}$ be a quasi contraction of type (B). Then, $\mathrm{T}$ has a unique fixed point in $\mathrm{X}$ provided that one of the following conditions holds:

(i) T is sequentially d-d-continuous;

(ii) $\mathrm{T}$ is sequentially $\mathrm{d}-\mathrm{d}^{-1}$-continuous;

(iii) Thas the following property:

$$
\inf \{d(y, x)+d(x, T x): x \in X\}>0
$$

for every $y \in X$ with $y \neq T y$.

Proof. Let $x_{0} \in X$ be arbitrary and $\left\{x_{n}\right\}$ be the Picard sequence, that is, $x_{n}=T x_{n-1}$ for all $n \in \mathbb{N}$. By Proposition 2.4, the sequence $\left\{x_{n}\right\}$ is a left K-Cauchy sequence in the quasi metric space $(X, d)$. Then, since $(X, d)$ is left $\mathcal{K}$-complete, there exists $z \in X$ such that $\left\{x_{n}\right\}$ d-converges to $z$, that is, $d\left(z, x_{n}\right) \rightarrow 0$ as $n \rightarrow \infty$.

Now, assume (i) holds. Since $T$ is sequentially $d$-d-continuous mapping, then

$$
\mathrm{d}\left(\mathrm{T} z, x_{\mathrm{n}}\right)=\mathrm{d}\left(\mathrm{T} z, \mathrm{~T} x_{\mathrm{n}-1}\right) \rightarrow 0 \text { as } \mathrm{n} \rightarrow \infty .
$$

Suppose $z \neq \mathrm{T} z$, then

$$
\begin{aligned}
0 & <d(z, T z) \leqslant d\left(z, x_{n+1}\right)+d\left(T x_{n}, T z\right) \\
& \leqslant d\left(z, x_{n+1}\right)+\lambda M_{B}\left(x_{n}, z\right) \\
& =d\left(z, x_{n+1}\right)+\lambda \max \left\{d\left(z, x_{n}\right), d\left(x_{n}, T x_{n}\right), d(z, T z), d\left(T z, x_{n}\right), d\left(z, T x_{n}\right)\right\} \\
& =d\left(z, x_{n+1}\right)+\lambda \max \left\{d\left(z, x_{n}\right), d\left(x_{n}, x_{n+1}\right), d(z, T z), d\left(T z, T x_{n-1}\right), d\left(z, x_{n+1}\right)\right\} .
\end{aligned}
$$

Letting $n \rightarrow \infty$, we get $0<d(z, T z) \leqslant \lambda d(z, T z)$, which is a contradiction. Therefore we have $z=T z$. By Proposition 2.6, the fixed point is unique. The proof is similar to proof of Theorem 2.8 when the other cases (ii) or (iii) hold.

Now we present similar results when the quasi metric space is left $\mathcal{M}-$-complete. However, we need the Hausdorffness condition in the case (i) in the following theorem.

Theorem 2.11. Let $(\mathrm{X}, \mathrm{d})$ be a left $\mathcal{N}$-complete $\mathrm{T}_{1}$-quasi metric space and $\mathrm{T}: \mathrm{X} \rightarrow \mathrm{X}$ be a quasi contraction of type $(A)$ or of type (B). Then, T has a unique fixed point in $\mathrm{X}$ provided that one of the following conditions holds:

(i) $\left(\mathrm{X}, \tau_{\mathrm{d}^{-1}}\right)$ is Hausdorff space $\mathrm{T}$ is sequentially $\mathrm{d}^{-1}-\mathrm{d}^{-1}$-continuous;

(ii) $\mathrm{T}$ is sequentially $\mathrm{d}^{-1}-\mathrm{d}$-continuous;

(iii) Thas the following property:

$$
\inf \{d(x, y)+d(x, T x): x \in X\}>0
$$

for every $\mathrm{y} \in \mathrm{X}$ with $\mathrm{y} \neq \mathrm{T} y$. 
Proof. Let $x_{0} \in X$ be arbitrary and $\left\{x_{n}\right\}$ be the Picard sequence, that is, $x_{n}=T x_{n-1}$ for all $n \in \mathbb{N}$. By Proposition 2.4, the sequence $\left\{x_{n}\right\}$ is a left K-Cauchy sequence in the quasi metric space. Since $(X, d)$ is left $\mathcal{M}$-complete, there exists $z \in X$ such that $\left\{x_{n}\right\} d^{-1}$-converges to $z$, that is, $d^{-1}\left(z, x_{n}\right)=d\left(x_{n}, z\right) \rightarrow 0$ as $n \rightarrow \infty$.

Now, we will consider the following three cases:

(i) Let $\left(X, \tau_{d^{-1}}\right)$ be Hausdorff space and $T$ be sequentially $d^{-1}-d^{-1}$-continuous mapping. Then

$$
\mathrm{d}^{-1}\left(\mathrm{~T} z, \mathrm{x}_{\mathrm{n}+1}\right)=\mathrm{d}^{-1}\left(\mathrm{~T} z, \mathrm{~T} x_{\mathrm{n}}\right) \rightarrow 0 \text { as } \mathrm{n} \rightarrow \infty .
$$

Since $\left(X, \tau_{d^{-1}}\right)$ is Hausdorff space, we get $z=T z$.

(ii) Let $T$ be sequentially $d^{-1}$-d-continuous. Then, since $d\left(x_{n}, z\right)=d^{-1}\left(z, x_{n}\right) \rightarrow 0$ as $n \rightarrow \infty$, we get $\mathrm{d}\left(\mathrm{T} z, \mathrm{~T} x_{\mathrm{n}}\right) \rightarrow 0$ as $\mathrm{n} \rightarrow \infty$. So, we have

$$
\mathrm{d}(\mathrm{T} z, z) \leqslant \mathrm{d}\left(\mathrm{T} z, T x_{n}\right)+\mathrm{d}\left(x_{\mathrm{n}+1}, z\right) \rightarrow 0 \text { as } \mathrm{n} \rightarrow \infty .
$$

Since $d$ is $T_{1}$-quasi metric, we get $z=T z$.

(iii) Let $T$ has a property that $\inf \{d(x, y)+d(x, T x): x \in X\}>0$ for every $y \in X$ with $y \neq T y$. Now, assume that $z \neq \mathrm{T} z$, then by our assumption, we obtain (by taking on account the left K-Cauchyness of $\left.\left\{x_{n}\right\}\right)$

$$
\begin{aligned}
0 & <\inf \{d(x, z)+d(x, T x): x \in X\} \\
& \leqslant \inf \left\{d\left(x_{n}, z\right)+d\left(x_{n}, T x_{n}\right): n \in \mathbb{N}\right\} \\
& =\inf \left\{d\left(x_{n}, z\right)+d\left(x_{n}, x_{n+1}\right): n \in \mathbb{N}\right\} \\
& =0,
\end{aligned}
$$

which is a contradiction. Hence $z=T z$.

\section{Acknowledgment}

The authors are thankful to the referees for making valuable suggestions leading to the better presentation of the paper.

\section{References}

[1] E. Alemany, S. Romaguera, On right K-sequentially complete quasi-metric spaces, Acta Math. Hungar., 75 (1997), 267-278. 1

[2] I. Altun, M. Olgun, G. Minak, Classification of completeness of quasi metric space and some new fixed point results, Nonlinear Funct. Anal. Appl., 22 (2017), 371-384. 1

[3] L. Ćirić, Fixed Point Theory Contraction Mapping Principle, Faculty of Mechanical Enginearing, University of Belgrade, Beograd, (2003). 2, 2

[4] S. Cobzaş, Functional analysis in asymmetric normed spaces, Birkhuser-Springer, Basel, (2013). 1

[5] H. Dağ, G. Minak, I. Altun, Some fixed point results for multivalued F-contractions on quasi metric spaces, Rev. R. Acad. Cienc. Exactas Fs. Nat. Ser. A Math. RACSAM, 111 (2017), 177-187. 1

[6] H. Dağ, S. Romaguera, P. Tirado, The Banach contraction principle in quasi-metric spaces revisited, Proceeding of the Workshop on Applied Topological Structures WATS'15, 25-31. 1

[7] J. C. Kelly, Bitopological spaces, Proc. London Math. Soc., 13 (1963), 71-89. 1

[8] H.-P. A. Künzi, Nonsymmetric distances and their associated topologies: About the origins of basic ideas in the area of asymmetric topology, Handbook of the history of general topology (Springer), 3 (2001), 853-968. 1

[9] I. L. Reilly, P. V. Subrahmanyam, M. K. Vamanamurthy, Cauchy sequences in quasi-pseudo-metric spaces, Monatsh. Math., 93 (1982), 127-140. 1

[10] S. Romaguera, A. Gutiérrez, A note on Cauchy sequences in quasi-pseudometric spaces, Glasnik Mat., 21 (1986), 191200.

[11] S. Romaguera, Left K-completeness in quasi-metric spaces, Math. Nachr., 157 (1992), 15-23. 1

[12] M. Sarwar, M. U. Rahman, G. Ali, Some fixed point results in dislocated quasi metric (dq-metric) spaces, J. Inequal. Appl., 2014 (2014), 11 pages. 1 